\title{
Complicaciones del tratamiento endoscópico del reflujo vesicoureteral en la infancia
}

\author{
A. Serrano Durba, M.A. Bonillo García, F. Moragues Estornell, C. Domínguez Hinarejos, \\ C. Sanguesa*, M. Martínez Verduch, F. García Ibarra
}

Servicio de Urología *Servicio de Radiodiagnóstico Infantil. Hospital La Fe. Valencia.

Actas Urol Esp 2006 (30): 170-174

\section{RESUMEN}

COMPLICACIONES DEL TRATAMIENTO ENDOSCÓPICO DEL REFLUJO VESICOURETERAL EN LA INFANCIA

Introducción: El reflujo vesicoureteral (RVU) afecta aproximadamente al $1 \%$ de los niños y constituye un factor de riesgo de lesión renal a largo plazo. En la última década los avances en este campo han demostrado que la inyección endoscópica subureteral de agentes inertes para corregir el RVU infantil, es una alternativa aceptada frente a la profilaxis antibiótica a largo plazo y a la cirugía abierta.

Objetivo: Determinar las complicaciones del tratamiento endoscópico del RVU en niños.

Material y Métodos: Realizamos un estudio retrospectivo de todos los pacientes con RVU sometidos a un tratamiento endoscópico en nuestro centro desde Enero de 1999 a Septiembre de 2003, con un total de 516 implantes submucosos de agentes inertes. Todos los pacientes fueron evaluados mediante ecografía como primera técnica de imagen diagnóstica, con la intención de determinar las complicaciones asociadas, fundamentalmente obstrucción ureteral y migración del material inyectado.

Resultados: De las 516 intervenciones, en 455 se utilizaron partículas de polidimetilsiloxano (Macroplastique ${ }^{\circledR}$ ), en 44 microesferas de dextrano (Deflux ${ }^{\circledR}$ ) y en 17 pasta de politetrafluoroetilo (Polytef ${ }^{\circledR}$ ). Se detectaron complicaciones en 10 pacientes (1,9\%) (5 niños y niñas), con un rango de edad entre 15 meses y 11,4 años, correspondientes a 8 implantes con Macroplastique ${ }^{\circledR}$ y a 2 con Deflux ${ }^{\circledR}$. Entre las complicaciones destacan 5 ureterohidronefrosis, de las que 2 precisaron derivación urinaria mediante nefrostomía, 3 migraciones del material implantado, 1 epididimitis y 1 edema vesical.

Conclusiones: La inyección endoscópica de agentes inertes se ha convertido en una alternativa segura a la cirugia abierta en el manejo del RVU infantil.

Palabras clave: Reflujo vesicoureteral. Tratamiento endoscópico. Complicaciones.

\section{ABSTRACT}

VESICOURETERIC REFLUX ENDOSCOPIC TREATMENT COMPLICATIONS IN CHILDHOOD

Introduction: Nowadays, around $1 \%$ of children suffer from vesicoureteral reflux (VUR), which represents a risk factor for long-term kidney damage. In the last decade, development in this field has shown that subureteric endoscopic injection of bulking agents for childhood VUR is an acceptable alternative unlike long-term antibiotic prophylaxis and open surgery.

Objective: To establish the complications of childhood VUR endoscopic treatment.

Material and Methods: A retrospective analysis was carried out for every patient treated endoscopically in our department from January 1999 to September 2003, with a total amount of 516 implants of submucous bulking agents. All patients were assessed with an ultrasound as a first diagnostic technique so as to establish the complications associated with it, especially ureteral obstruction and injected agent migration.

Results: Out of 516 cases, Macroplastique ${ }^{\circledR}$ was used in 455, Deflux® in 44 and Polytef® in 17.10 patients (1.9\%) (5 girls / 5 boys), with ages ranging from 15 months to 11,4 years, presented some kind of complications (Macroplastique ${ }^{\circledR}$ was used in 8 cases and Deflux ${ }^{\circledR}$ in 2). Among them, 5 were remarkable due to an ureterohydronefrosis, 2 of which needed a nephrostomy. 3 patients presented an implant migration, 1 epididymitis and 1 bladder edema.

Conclusions: Endoscopic bulking agent injection has become a safe alternative to open surgery in childhood VUR management.

Keywords: Vesicoureteral reflux. Endoscopic treatment. Complications. 
$\mathrm{E}$ reflujo vesicoureteral (RVU) es una entidad frecuente en la infancia que predispone a un lesión renal a largo plazo (insuficiencia renal e hipertensión), cuando coexiste con infecciones urinarias de repetición (ITU) ${ }^{1-4}$. El tratamiento del RVU se realiza con la intención de prevenir este daño del parénquima renal y sus consecuencias futuras. Actualmente, se puede optar tanto una actitud conservadora como quirúrgi$\mathrm{ca}^{5}$. El tratamiento médico implica una profilaxis antibiótica a largo plazo para evitar la aparición de ITU mientras persiste el reflujo, fundamentado en la resolución espontánea del RVU en el 15$40 \%$ de los casos en función del grado ${ }^{6}$. Sin embargo, aunque este planteamiento obvia la cirugía, tiene la desventaja de la difícil cumplimentación y el desarrollo de resistencia antibióti$\mathrm{ca}^{7}$, y lo que es aún más preocupante, incluso realizando una correcta profilaxis, algunos trabajos han determinado que la incidencia de lesión renal en esta población se comprende entre el $4.7-23 \%{ }^{8}$. Por otro lado, aunque el reimplante ureteral mediante cirugía abierta alcanza en la mayoría de series un éxito del prácticamente $95 \%{ }^{9}$, su indicación se suele reservar para aquellos casos donde la severidad del reflujo compense el riesgo quirúrgico. Además, esta intervención quirúrgica no está exenta de complicaciones incluso cuando la realiza un cirujano experto, así en el informe de la AUA la tasa de obstrucciones tras el reimplante ureteral que requirieron una reintervención fue de $0,3-9,1 \%{ }^{10}$.

El tratamiento endoscópico, que surgió al principio de $1980^{11}$, consiste en la inyección de una sustancia en la pared vesical por debajo del orificio ureteral con la finalidad de alargar la porción intramural del uréter, y prevenir así el reflujo de orina al tracto urinario superior. Desde su instauración como una alternativa válida al tratamiento clásico se han investigado muchos agentes sin encontrar todavía el material ideal, ya que la preocupación por la seguridad y la eficacia de los mismos ha limitado su uso generalizado ${ }^{12}$.

El objetivo que ha motivado este estudio es determinar las complicaciones del tratamiento endoscópico del RVU en niños y objetivar así si la inyección subureteral de agentes inertes constituye una alternativa terapéutica segura a la cirugia abierta.

\section{MATERIAL Y MÉTODO}

En el periodo entre 1999-2003 se trataron endoscópicamente 516 unidades ureterales, de todo grado, en un grupo de 349 pacientes (223 niñas/126 niños) en el servicio de Urología Infantil del Hospital La Fe de Valencia. En el momento del procedimiento los pacientes tenían una edad media de 7,18 años. (15m-16a). Los RVU de grado I solamente se trataron en pacientes con RVU contralateral de mayor grado o en niñas prepúberes. En 455 procedimientos se utilizó partículas de polidimetilsiloxano (Macroplastique ${ }^{\circledR}$ ), en 44 microesferas de dextrano $\left(\right.$ Deflux $\left.x^{\circledR}\right)$ y en 17 pasta de politetrafluoroetilo (Polytef). Se incluyeron en el estudio tanto RVU primarios como secundarios, sin encontrar diferencias estadísticamente significativas en cuanto al volumen de material inyectado ( $p=0,167)$ para lo cual aplicamos la prueba de Chi-cuadrado $\left(\mathrm{Chi}^{2}\right)$, que nos permite realizar una "prueba de bondad de ajuste", es decir, contrastar si las frecuencias observadas en cada una de las clases de una variable categórica (tipo de RVU) varían de forma significativa de las frecuencias que se esperaría encontrar si la muestra hubiese sido extraída de una población con una determinada distribución de frecuencias. En los RVU primarios se utilizó una media de $0,72 \mathrm{ml}$, mientras en los secundarios una media de $0,81 \mathrm{ml}$. Todos los pacientes fueron evaluados mediante ecografía al mes de la intervención con la intención de determinar las complicaciones asociadas, y eran controladas clínicamente cada 6 meses durante un seguimiento mínimo de 12 meses.

\section{RESULTADOS}

De los 516 procedimientos, tan solo se objetivaron 10 situaciones clínicas $(1,9 \%)$ que se pudieran catalogar como complicaciones. No hubo diferencias entre ambos sexo ( 5 niños/ 5 niñas) ni entre las diferentes edades en la que se presentasen en mayor número (rango de edad entre 15meses y 11,4 años). En cuanto al material utilizado, en 8 ocasiones se había empleado Macroplastique ${ }^{\circledR}$ y en 2 Deflux ${ }^{\circledR}$, no siendo esta diferencia estadísticamente significativa $\left(\mathrm{Chi}^{2}, \mathrm{p}=0,18\right)$; no se objetivó ninguna complicación en los pocos pacientes donde se uso Polytef ${ }^{\circledR}$ (Tabla 1). 
Tabla 1

Esquema de las complicaciones detectadas en el seguimiento de los pacientes con RVU tratados mediante inyección de agentes inertes

\begin{tabular}{|c|c|c|c|c|c|}
\hline Caso & Sexo & Edad & Material & Tiempo & Complicación \\
\hline 1 & q & 7 años & polidimetilsiloxano & 8 meses & RAO \\
\hline 2 & q & 11 años & polidimetilsiloxano & 24 horas & Ectasia Pielocalicial \\
\hline 3 & $0^{x}$ & 15 meses & dextranómero $\mathrm{A} / \mathrm{H}$ & 48 horas & Ectasia Pielocalicial \\
\hline 4 & q & 7 años & polidimetilsiloxano & 24 horas & Migración calicial \\
\hline 5 & $\sigma^{\pi}$ & 4 años & polidimetilsiloxano & 37 días & Ectasia Pielocalicial $(*)$ \\
\hline 6 & q & 8 años & polidimetilsiloxano & 16 días & Ectasia Pielocalicial (*) \\
\hline 7 & $0^{7}$ & 6 años & dextranómero $\mathrm{A} / \mathrm{H}$ & 28 días & Ectasia Pielocalicial \\
\hline 8 & $\sigma^{x}$ & 5 años & polidimetilsiloxano & 45 días & Migración vesical \\
\hline 9 & $\sigma^{x}$ & 4 años & polidimetilsiloxano & 4 días & Epididimitos Aguda \\
\hline 10 & q & 6 años & polidimetilsiloxano & 25 días & Hematuria Autolimitada \\
\hline
\end{tabular}

(*) nefrostomía percutánea urgente y reimplante ureteral programado.

RAO: retención aguda de orina

Entre las complicaciones destacamos la ectasia ureteropielocalicial mayor que la que presentaba el paciente previo a la intervención, registrada en 5 pacientes; 2 de los cuales no presentaron sintomatología alguna y fue un hallazgo ecográfico casual (Fig. 1). Sin embargo, 3 pacientes habían sufrido una clínica compatible con crisis renoureteral durante el seguimiento a los 37,16 y 28 días del procedimiento. En estos 2 pacientes fue necesaria la realización de una nefrostomía percutánea de forma urgente tras intento infructuosos de cateterismo, y como solución definitiva un reimplante ureteral programado según la técnica descrita por Cohen. La ectasia del resto de los pacientes se resolvió espontáneamente en el transcurso del seguimiento clínico.

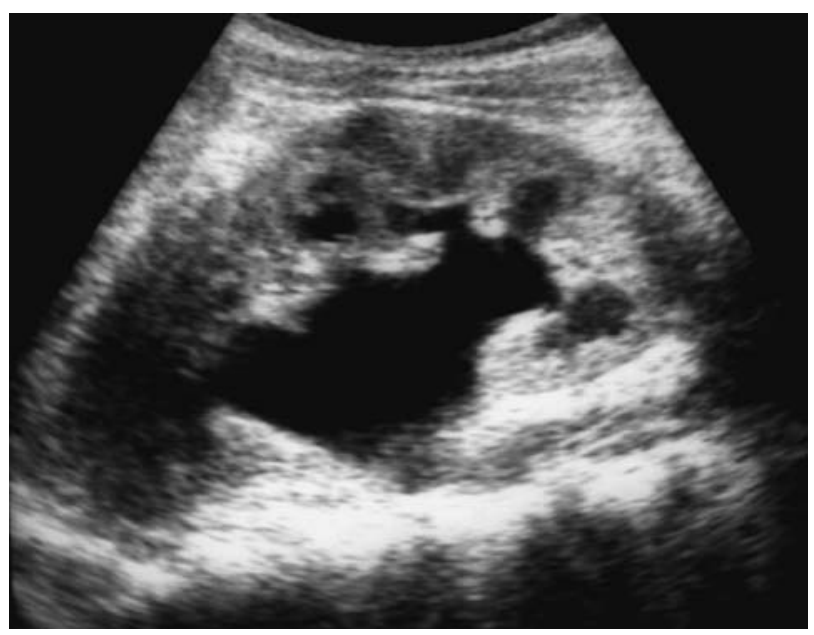

FIGURA 1
En 3 pacientes se constató la migración del material inyectado. Una paciente debutó con un cuadro de retención aguda de orina (RAO) a los 8 meses del procedimiento y a la exploración se evidenció la presencia de material calcificado impactado en el meato uretral que extrajo mediante pinzas (Fig. 2). En otro paciente se localizaron restos de considerable tamaño en el grupo calicial medio y superior (Fig. 3) a los 45 días en el contexto de molestias lumbares inespecíficas, que se expulsaron a las pocas semanas sin ninguna dificultad, $\mathrm{y}$ en un último, el habón se desplazó por la submucosa medialmente, recidivándo así el RVU. En cuanto al resto de complicaciones destacamos un cuadro de hematuria autolimitada a los 25 días secundario a un edema vesical (Fig. 4) y una epididimitis aguda al $4^{\circ}$ día, ambas mejoraron $\sin$ necesidad de ninguna actitud especial.

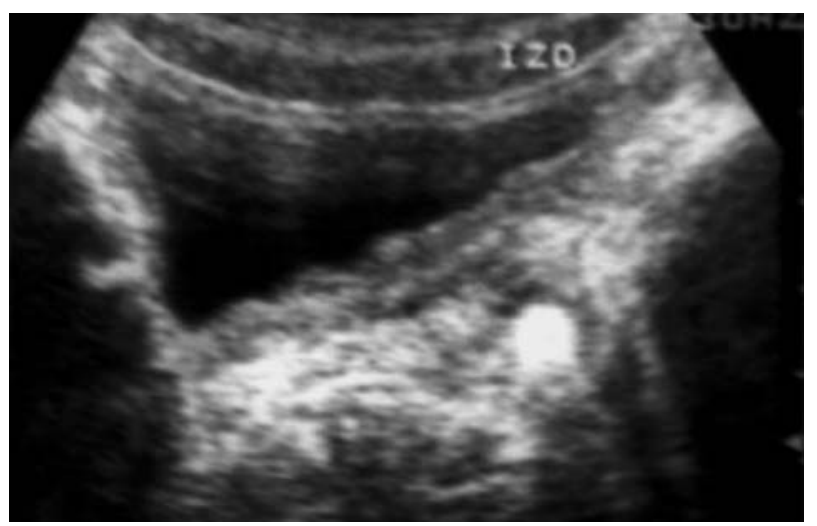

FIGURA 2 




FIGURA 3

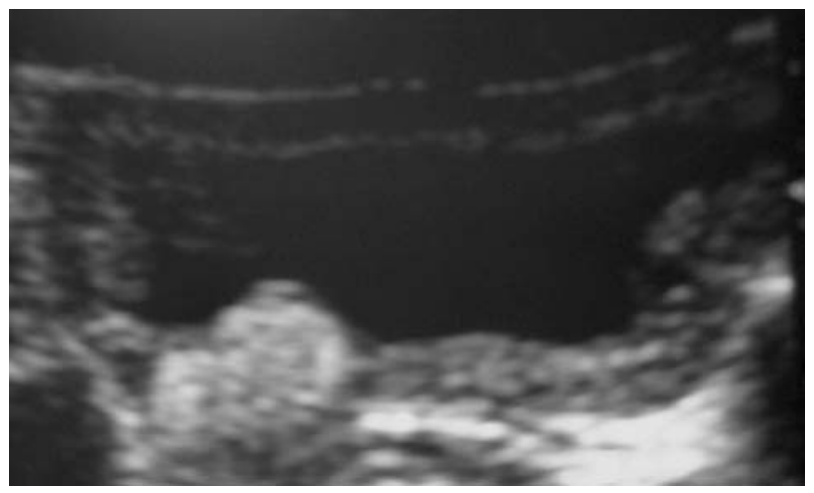

FIGURA 4

De una forma generalizada, estas complicaciones no se asociaron de forma significativa ni con el volumen inyectado $(p=0,704)$ ni tampoco con el resultado obtenido $(p=0,657)$, al igual que tampoco hubo una relación estadísticamente significativa entre el volumen inyectado y el éxito del procedimiento $(p=0,361)$.

\section{DISCUSIÓN}

El debate acerca de cuál es el agente inerte ideal en el tratamiento endoscópico del RVU infantil sigue siendo controvertido. Esta sustancia debería ser efectiva, segura y estable con el paso del tiempo, es decir que la pérdida de volumen sea mínima y que no migre o se extruya fuera de la vejiga. A su vez este material ha de ser biocompatible, no carcinogénico, ni antigénico. En este sentido, el politetrafluoretileno ha sido el agente más utilizado que se describe en la literatura y a pesar de alcanzar un tasa de curación cercana al $85 \%^{13}$, dado el tamaño de las partículas ( $>90 \%$ son menores de $40 \mu)$ se han descrito casos de embolización a distancia en otros órganos ${ }^{14}$. En nuestra serie no hemos objetivado ninguna reacción adversa en los 17 pacientes en los que utilizamos politetrafluoretileno.

La utilización del colágeno ha conseguido una tasa de curación que varía según publicaciones del $62-83 \%^{15,16}$. Su principal inconveniente es la necesidad de someter al paciente a una prueba cutánea previa al proceder de una proteína bovina que puede desencadenar una respuesta humoral y celular. De igual modo, su poca estabilidad en el tiempo debido a su biodegradación hace necesaria una reintervención por recurrencia del RVU, a pesar de los buenos resultados iniciales ${ }^{17}$. Este mismo inconveniente lo presentan el resto de materiales autólogos ya sea grasa, condrocitos o células musculares de la vejiga, que además precisan de cultivos celulares que incrementan la morbilidad asociada a este procedimiento.

Estudios previos indican una tasa de éxito con el polidimetilsiloxano de $81-93 \%{ }^{18,19}$ al igual que advierten el riesgo de reacciones autoinmunes y malignización a largo plazo, lo cual ha sido demostrado en animales ${ }^{20}$. Además, dado que el tamaño de las partículas es $<80 \mu$ el riesgo de migración a distancia aumenta. Sin embargo, Herz et al. ${ }^{19}$, concluye en su estudio que la utilización de esta sustancia es segura con baja morbilidad asociada, ya que no se desarrolló ninguna ectasia pielocalicial de novo durante el seguimiento ni reacción alérgica en ninguno de los niños, al igual que tampoco objetivó ninguna migración del implante. Tan sólo un paciente $(1,4 \%)$ desarrolló una ITU febril que se resolvió ambulatoriamente con la toma de antibióticos. En nuestra serie, de los 455 procedimientos en 308 pacientes donde utilizamos polidimetilsiloxano $8(2,6 \%)$ presentaron algún tipo de complicación, y tan sólo 2 pacientes $(0,65 \%)$ precisaron derivación urinaria urgente por uropatía obstructiva, tasa menor que la publicada en otras series con este mismo material ${ }^{21}$.

En 1995, Stenberg y Lackgren ${ }^{22}$ incluyen un nuevo biomaterial basado en el ácido hialurónico y dextranómero, que alcanza una tasa de cura- 
ción del $75 \%$. En un principio, el volumen del implante no se reduce con el tiempo debido al crecimiento de los fibroblastos en su interior y a la producción de colágeno endógeno entre las microsferas, así Kirsch y cols. ${ }^{23}$ encuentran una disminución del tamaño del habón menor del $20 \%$ al año de seguimiento. Del mismo modo, dado el gran tamaño de sus microsferas (80-25 $\mu$ ) es improbable su migración de la vejiga hacia otras regiones del organismo. Al igual que en las distintas series publicadas ${ }^{23-25}$, en nuestro estudio no se han objetivado complicaciones importantes relacionadas con este material ni con el procedimiento realizado.

\section{CONCLUSIÓN}

La inyección endoscópica de agentes inertes es una alternativa segura en el tratamiento del RVU infantil de cualquier etiología, con escasas complicaciones de baja entidad e independientes del tipo de sustancia utilizada.

\section{REFERENCIAS}

1. Lenaghan D, Whitaker JG, Jensen F, Stephens FD. The natural history of reflux and long-term effects of reflux on the kidney. J Urol 1976;115(6):728-730.

2. Bailey RR. Vesico-ureteric reflux and reflux nephropathy. Kidney Int Suppl 1993;42:S80-85.

3. Jacobson SH, Hansson S, Jakobsson B. Vesico-ureteric reflux: occurrence and long-term risks. Acta Paediatr Suppl 1999;88(431):22-30.

4. Riccabona M. Management of recurrent urinary tract infection and vesicoureteral reflux in children. Curr Opin Urol 2000; $10(1): 25-28$.

5. Medical versus surgical treatment of primary vesicoureteral reflux: report of the International Reflux Study Committee. Pediatrics 1981;67(3):392-400.

6. Van Capelle JW, de Haan T, El Sayed W, Azmy A. The longterm outcome of the endoscopic subureteric implantation of polydimethylsiloxane for treating vesico-ureteric reflux in children: a retrospective analysis of the first 195 consecutive patients in two European centres. BJU Int 2004;94 (9):13481351.

7. Bollgren I. Antibacterial prophylaxis in children with urinary tract infection. Acta Paediatr Suppl 1999;88(431):48-52.

8. Connolly LP, Treves ST, Connolly SA, Zurakowski D, Share JC, Bar-Sever Z, et al. Vesicoureteral reflux in children: incidence and severity in siblings. J Urol 1997;157(6): $2287-$ 2290.

9. Elder JS. Guidelines for consideration for surgical repair of vesicoureteral reflux. Curr Opin Urol 2000;10(6):579-585.

10. Elder JS, Peters CA, Arant BS, Jr., Ewalt DH, Hawtrey CE, Hurwitz RS, et al. Pediatric Vesicoureteral Reflux Guidelines Panel summary report on the management of primary vesicoureteral reflux in children. J Urol 1997;157 (5):1846-1851.
11. Matouschek E. [Treatment of vesicorenal reflux by transurethral teflon-injection (author's transl)]. Urologe A 1981; 20(5):263-264.

12. Stenberg A, Hensle TW, Lackgren G. Vesicoureteral reflux: a new treatment algorithm. Curr Urol Rep 2002;3(2):107-114.

13. Puri P. Ten year experience with subureteric Teflon (polytetrafluoroethylene) injection (STING) in the treatment of vesicoureteric reflux. Br J Urol 1995;75(2):126-131.

14. Sugiyama T, Hanai T, Hashimoto K, Umekawa T, Kurita T. Long-term outcome of the endoscopic correction of vesico-ureteric reflux: a comparison of injected substances. BJU Int 2004;94(3):381-383.

15. Frey P, Gudinchet F, Jenny P. GAX 65: new injectable crosslinked collagen for the endoscopic treatment of vesicoureteral reflux-a double-blind study evaluating its efficiency in children. J Urol 1997;158(3 Pt 2):1210-1212.

16. Reunanen M. Correction of vesicoureteral reflux in children by endoscopic collagen injection: a prospective study. J Urol 1995; 154(6):2156-2158.

17. Haferkamp A, Contractor H, Mohring K, Staehler G, Dorsam J. Failure of subureteral bovine collagen injection for the endoscopic treatment of primary vesicoureteral reflux in longterm follow-up. Urology 2000;55(5):759-763.

18. Dodat H, Valmalle AF, Weidmann JD, Collet F, Pelizzo G, Dubois R. [Endoscopic treatment of vesicorenal reflux in children. Five-year assessment of the use of Macroplastique]. Prog Urol 1998;8(6):1001-1006.

19. Herz D, Hafez A, Bagli D, Capolicchio G, McLorie G, Khoury A. Efficacy of endoscopic subureteral polydimethylsiloxane injection for treatment of vesicoureteral reflux in children: a North American clinical report. J Urol 2001;166(5):1880-1886.

20. Hatanaka S, Oneda S, Okazaki K, Shong LJ, Yoshida A, Isaka $\mathrm{H}$, et al. Induction of malignant fibrous histiocytoma in female Fisher rats by implantation of cyanoacrylate, zirconia, polyvinyl chloride or silicone. In Vivo 1993;7(2):111-115.

21. Al-Hunayan AA, Kehinde EO, Elsalam MA, Al-Mukhtar RS. Outcome of endoscopic treatment for vesicoureteral reflux in children using polydimethylsiloxane. J Urol 2002;168 (5):2181-2183.

22. Stenberg A, Lackgren G. A new bioimplant for the endoscopic treatment of vesicoureteral reflux: experimental and shortterm clinical results. J Urol 1995;154(2 Pt 2):800-803.

23. Kirsch AJ, Perez-Brayfield MR, Scherz HC. Minimally invasive treatment of vesicoureteral reflux with endoscopic injection of dextranomer/hyaluronic acid copolymer: the Children's Hospitals of Atlanta experience. J Urol 2003; 170(1):211-215.

24. Lackgren G, Wahlin N, Skoldenberg E, Neveus T, Stenberg A. Endoscopic treatment of vesicoureteral reflux with dextranomer/hyaluronic acid copolymer is effective in either double ureters or a small kidney. J Urol 2003;170(4 Pt 2):1551-1555; discussion 1555.

25. Puri P, Chertin B, Velayudham M, Dass L, Colhoun E. Treatment of vesicoureteral reflux by endoscopic injection of dextranomer/hyaluronic Acid copolymer: preliminary results. J Urol 2003;170(4 Pt 2):1541-1544; discussion 1544.

Dr. M.A. Bonillo García

E-mail: miggs89@yahoo.es

(Trabajo recibido 24 de mayo 2005) 